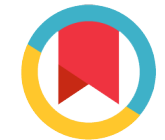

Check for updates
*For correspondence:

bbarjasteh@gmail.com

Competing interests: The authors declare that no competing interests exist.

Received: 28 January 2017

Accepted: 27 June 2017

Published: 30 June 2017

Copyright The Author(s) 2017. This article is published with open access by BioMedPress (BMP).

This article is distributed under the terms of the Creative Commons Attribution License (CC-BY 4.0) which permits any use, distribution, and reproduction in any medium, provided the original author(s) and the source are credited.

\section{The impact of 8 weeks selected corrective exercises on neck pain, range of motion in the shoulder and neck of lifesaver women who suffering from forward head posture and myofascial pain syndrome}

\section{Melody Tabatabaei ${ }^{1}$, Behrouz Barjasteh Mohebbi ${ }^{2,{ }^{*}}$, Alireza Rahimi ${ }^{1}$}

${ }^{1}$ Department of Physical Education and Sport Sciences, Karaj Branch, Islamic Azad University, Karaj, Iran

${ }^{2}$ Department of Physical Education, Iran University of Science and Technology (IUST), Narmak, Tehran

\section{Abstract}

Background: The purpose of this research was studying the impact of 8 weeks selected corrective exercises on neck pain, range of motion in the shoulder and neck of lifesaver women who suffering from forward head posture and myofascial pain syndrome. Methods: The method of research is semi-experimental. The population consists of 30 lifesaver women who suffering from forward head posture and myofascial pain syndrome who they placed randomly in two groups of experimental (33 \pm 2.2$)$ and control $(33 \pm 2.5)$. Research plan was as the pre-test and post-test with control group. The exercise protocol was carried out by experimental groups for 8 weeks, 3 sessions per week, each session lasting 45 minutes. Studied variables include myofascial pain in the neck, shoulder and neck range of motion. The mean and standard deviation were used as descriptive statistics and in the section of inferential statistics analysis of covariance was used. Results: Results of research indicated that, the protocol on the reform exercise of neck pain $(P=0.001)$, range of motion of shoulder joint $(P=0.001)$ and neck range of motion ( $P=0.001)$ has significant difference. Conclusion: Therefore, lifesavers women can benefit from it as a training program to improve and prevent damage caused by head forward and myofascial pain syndrome.

\section{Keywords}

Myofascial Neck Pain, Range of Motion, Trapezius Muscle 


\section{Introduction}

In many parts of the body, there are receptors that stimulate them, transferring unpleasant emotions as pain from the related organ to the brain. The human brain interprets pain and attempts to get rid of the unpleasant feeling of pain (Alter, 1996). To create pain it is necessary to stimulate receptors that are present in many parts of the body. The stimulators of pain are divided in two chemical and physical groups. For example, when our hands are burning, chemical stimuli are active and when the pain signal arrives at the hand, physical receivers are responsible for that (Luo et al., 2004). Chronic pain is and continues to be one of the major medical problems throughout the world. Annually millions of human beings suffer from chronic pain but, unfortunately, many patients do not receive proper treatment.

Chronic pain is the most common cause of human suffering and disability across the world, and seriously affects quality of human life (Shahi moridi, 2009). Research studies have shown that today musculoskeletal pain has become more common than 40 years ago. Muscles as dynamic factors have a leading role in the movement and activities of daily living. Muscle tissues are more exposed to small ruptures than the body during daily activities and experience pain. Therapists have been focusing more attention during patient examinations on interpretation of pain in the bones, joints, and peripheral and central nervous systems.

Muscle trigger points are commonly seen in humans. These points appear as a tightened band in skeletal muscle fibers and manifest as pain and hypersensitivity. Pain in these areas can be released to the outer regions and lead to local extension responses. Complex theories have been suggested about bad performance and muscle pain. One of the key components of these theories is energy crisis, considered to be the pathophysiology of the muscle trigger points. These theories argue that factors such as increased muscle and prolonged release of calcium, increased metabolic activity, local ischemia and vascular release of active substances can all lead to the creation of a defect cycle (Hanten et al., 2000).

All people in life have experienced some sort of pain. Muscle tissue consists of $40 \%$ to $50 \%$ of body weight and almost $85 \%$ of people in their lifetime will complain of muscle aches (Amanolahi Asadollah, 2009). Myofascial pain syndrome is the most common cause of skeletal muscle pain, with trigger points originating from one or more connective tissues about $30-70 \%$ of the time, as reported in various studies (Schleip, 2003). Trigger points can be referred to as pain in a special point of skeletal muscle or fascia muscle which is sensitive to touch such that exertion of pressure on that point can lead to sensitivity or reflective pain (Hou et al., 2002).

The advent of pain with origin of trigger points in most patients can be associated with spasms, stiffness, sensitivity to pressure, muscle weakness, 
limitation of movement, and limited range of motion in the neck and upper back (trapezius muscle). The cause of this pain is still not fully understood (Timmons and Ley, 1994). However, some general reasons for pain are pressure caused by frequent contractions, small repetitive blows, stagnant and limited body movement in administrative jobs, poor body posture, lack of exercise, and stress. The above factors can all cause pain, regardless of the originating muscle pain. Generally, pain is observed more in women than men because of muscle weakness (Salari, 2009).

Pain associated with limitations in performance and decreased range of motion can occur in almost all muscles of the body (Ghiasi et al., 2008). Trigger points are present in all parts of the body but particularly for the trapezius muscles, given their role in stabilization and support and their frequent use (Naroii et al., 2010). Often, according to life circumstances, people are put in situations where the muscles are shortened or stretched. Immobilization affects the structure and function of the muscles. The repetitive micro-trauma leads to an increase of the pressure on the muscle fibers and trigger points. The area of chronic pain will eventually show a decreased range of motion of the related muscles (Shahi moridi, 2009).

Any type of damage to the body tissue will cause inflammation which will, in turn, activate pain receptors in the body and begin a protective mechanism to increase muscle tension, leading to muscle spasms. Such muscle spasms are not like leg cramps. As a result of spasm, adhesions begin to take shape in the connective tissue. The adhesion leads to weakness and inelastic matrix which will result in reduced elasticity of soft tissue. The final result is change of tension length (which leads to reciprocal inhibition change), change of connections of pair power (which impacts excellence performance of partner muscles), and malfunction in Artrokintic performance (which causes change in motion of joints). If does not pay attention to these bonds, they can lead to the formation of permanent structural changes in soft tissues (Hodi et al., 2010). Consequences of incorrect posture can be so extensive, resulting in a negative impact on physical, psychological, economic and social aspects.

So far, many methods have been used to treat trigger points; these include ice massage, retching to make muscles active, and acupuncture or laser therapy (more or less for pain relief) (Roach et al., 2013). Today, corrective movements are considered as a branch of Physical Education Science and part of a noninvasive, low-risk, low-cost and yet highly enjoyable and refreshing solution to improve pain caused by trigger points (Kumar et al., 2015). Maintaining physical function is necessary to perform self-care activities in individuals with chronic conditions such as pain with origin of trigger points. In this regard, doing exercises regularly and improving physical strength can reduce the severity of pain and fatigue, while leading to an increased sense of confidence (Lundeberg et al., 1984). 
The recommended protocol includes treatment movements, stretching and resistance training. On one hand, these exercises help by removing waste materials in the tissues, leading to reduced pain and discomfort (the effect of increasing blood circulation will reduce complications such as muscle soreness). On the other hand, these exercises raise the pain threshold for nerve receptors since the treatment movements gradually add to the intensity of activity (in essence, the sensitivity of pain receptors decreases and the pain threshold increases) (Timmons and Ley, 1994). Pain and discomfort during the stretch run will lessen, while pain tolerance from strength exercises will increase and elongation tissues may possibly increase in length. Muscle stretching techniques also cause an increase in muscle flexibility and range of motion (Hanten et al., 2000). Doing some stretching movements may reduce treatment costs and complications derived from shortness of muscle tissues and prevent workforce disruptions and absences caused by muscle pain (Kumar et al., 2015).

Pharmacological treatment of trigger points only has a soothing role and cannot eliminate the underlying cause of the disorder (Lundeberg et al., 1984). On the contrary, physical therapy and massage therapy reduce pain and can restore normal muscle function as well (Hanten et al., 2000). The other treatment advantages are that they are less risky and have higher security and also their costs are less. Thus it seems that using such regular programs can have a major share in reducing effects of pain caused by trigger points, reduce the amount of drug use, mental and spiritual health of the patients. according to high prevalence of trigger pain among musculoskeletal pain and side effects such as headache, dizziness and neck pain, muscle stiffness, decreased range of motion and insomnia that followed the pain of trigger points and given that the impact of therapy on pain of trigger movements in a form of movement therapy has not been specifically investigated. Therefore, doing a study with the purpose of study the impact of selected corrective exercises protocol on the shoulder and neck pain, neck range of motion in the upper trapezius muscle lifesavers women with the forward head posture syndrome is necessary. Lifesavers because of the stress caused by stress and repetitive movements of the muscles involved in this job are one of the potential options to cause pain with trigger points. Understanding this issue that incorrect postures and repetitive movements can cause malfunction of the connective tissue system of human movement, physical fitness is essential for health professionals. So in this research, researchers are trying to answer the question that Is the protocol selected corrective exercises leads to decrease pain and increase range of motion in the shoulder and neck lifesavers forward head and myofascial pain syndrome? If yes to these questions, the reform as a non-invasive method used to reduce pain and correct deformities. 


\section{Materials-Methods}

The purpose of this research was studying the impact of 8 weeks selected corrective exercises on neck pain, range of motion in the shoulder and neck of lifesaver women suffering from forward head posture and myofascial pain syndrome. The method of research is semi-experimental. The population consisted of 30 people of lifesaver women suffering from forward head posture and myofascial pain syndrome which they place randomly in two groups of experimental age $(33 \pm 2.2)$ and control $(33 \pm 2.5)$. Research plan was as the pretest and post-test with control group. The exercise protocol was carried out by experimental groups for 8 weeks, 3 sessions per week, each session lasting 45 minutes. Studied variables include myofascial pain in the neck, shoulder and neck range of motion. The mean and standard deviation were used as descriptive statistics and in the section of inferential statistics analysis of covariance was used.

\section{The protocol of selected corrective exercises}

In this research according to previous studies the protocol of corrective exercises of forward head posture (NASM) was used as the protocol of selected corrective exercises. This protocol consists of four parts of the inhibitory techniques; stretching techniques, techniques activation and integration techniques that was conducted duration of 45 minutes in each stage of the exercise (10 minutes with exercise movements dedicated to warm up was 30 minutes of exercise was based on a research protocol and the 5-minute was done for recovery).the protocol of corrective exercises designed by the researcher according to the chain basics principle of national Academy of Sports Medicine training of America and after consultation with the consultants and supervisors was applied on participants. Selected protocol was applied on the experimental group and the control group did not conduct any activity related to the corrective exercises when the other group did activities and the implementation of corrective exercises.

\section{Results}

The mean, standard deviation, minimum and maximum gathering demographic characteristics including age, height, weight and experience are presented in Table 1. To test this hypothesis, analysis of covariance was used to effect corrective exercise on women with neck pain control and experimental groups specified. The results of this test are given in Table 2 .

As can be seen in Table 2, the results of analysis of covariance showed that there are statistically significant differences between the two groups $(p=0.001)$ therefore the hypothesis of research is confirmed as it can be seen partial eta 
squared also shows that more than 0.32 variance is explained by the difference between the two groups. The test statistic also shows that the pretest scores had no significant effect on the post-test scores $(p=0.852)$. To test this hypothesis, analysis of covariance was used to determine the effect of corrective exercise on the extent of the neck range of women in both experimental and control groups. The results of this test are given in Table 3 .

Table 1. Descriptive information of participants in the experimental and control group

\begin{tabular}{|l|l|c|c|c|c|c|}
\hline Group name & Variable & $\mathbf{n}$ & mean & SD & min & max \\
\hline $\begin{array}{l}\text { Experimental } \\
\text { group }\end{array}$ & age & 15 & 33.93 & 2.2 & 32 & 40 \\
\cline { 2 - 7 } & length & 15 & 165.6 & 2.9 & 157 & 170 \\
\cline { 2 - 7 } & weight & 15 & 67.2 & 3.7 & 60 & 74 \\
\hline Experience & 15 & 11 & 2.7 & 6 & 15 \\
\hline group & age & 15 & 33.73 & 2.5 & 30 & 38 \\
\hline & length & 15 & 166.4 & 1.8 & 164 & 171 \\
\hline & weight & 15 & 67.07 & 5.3 & 54 & 80 \\
\hline & Experience & 15 & 11.9 & 3.8 & 6 & 20 \\
\hline
\end{tabular}

Table 2. The results of analysis of covariance to compare the two groups in variable of pain level

\begin{tabular}{|l|c|c|c|c|c|c|}
\hline Source & SS & Df & MS & F & Sig. & Partial Eta squared \\
\hline Pretest & 0.058 & 1 & 0.058 & 0.035 & 0.852 & 0.001 \\
\hline $\begin{array}{l}\text { Experimental and } \\
\text { Control Group }\end{array}$ & 20.88 & 1 & 20.88 & 12.79 & 0.001 & 0.321 \\
\hline Error & 44.07 & 27 & 1.63 & & & \\
\hline * significance level a> 0.05 & & & & \\
\hline
\end{tabular}

As can be seen in Table 3, the results of analysis of covariance showed that there are statistically significant differences between the two groups $(p=0.001)$. So the hypothesis is confirmed. As can be seen, partial eta squared also shows that more than 0.49 variance is explained by the difference between the two groups. The test statistic also shows that the pretest scores had no significant effect on the post-test scores $(p=0.315)$. To test this hypothesis, analysis of covariance was used to test to be determined the effect of corrective exercises on the shoulder range of motion of women in both experimental and control groups. The results of this test are given in Table 4. 
Table 3. The results of analysis of covariance to compare the two groups in the variable of range of neck movement

\begin{tabular}{|l|c|c|c|c|c|c|}
\hline Source & SS & Df & MS & F & Sig. & Partial eta squared \\
\hline Pretest & 61.82 & 1 & 64.52 & 1.12 & 0.315 & 0.041 \\
\hline $\begin{array}{l}\text { Experimental } \\
\text { and Control } \\
\text { Group }\end{array}$ & 1813.8 & 1 & 1713.5 & 28.67 & 0.001 & 0.492 \\
\hline Error & 1541.5 & 27 & 59.54 & & & \\
\hline
\end{tabular}

As can be seen in Table 4, the results of analysis of covariance showed that there are statistically significant differences between the two groups $(p=0.001)$. So the hypothesis is confirmed. As can be seen, partial eta squared also shows that more than 0.61 variance is explained by the difference between the two groups. The test statistic also shows that the pretest scores had no significant effect on the post-test scores $(p=0.765)$. To test this hypothesis, analysis of covariance was used to test to be determined the effect of corrective exercises on the shoulder range of motion of women in both experimental and control groups.

Table 4. The results of analysis of covariance to compare the two groups in the shoulder flexion

\begin{tabular}{|l|c|c|c|c|c|c|}
\hline Source & SS & Df & MS & F & Sig. & $\begin{array}{c}\text { Partial eta } \\
\text { squared }\end{array}$ \\
\hline Pretest & 5.15 & 1 & 4.05 & 0.089 & 0.765 & 0.003 \\
\hline $\begin{array}{l}\text { Experimental } \\
\text { and Control } \\
\text { Group }\end{array}$ & 1894.4 & 1 & 2054.8 & 41.75 & 0.001 & 0.619 \\
\hline Error & 1247.01 & 27 & 41.6 & & & \\
\hline
\end{tabular}

\section{Discussion}

Results showed that selected corrective exercises have impact on neck pain intensity of lifesavers women who suffering from forward head posture and pain myofascial syndrome that probably its reason was reduction in trigger points in some neck muscles including sternocleidomastoid, corner tissues and upper trapezius muscles. Results of this study are consistent with results of Kumar et al (2015), Hanten et al (2000) and inconsistent with findings of Lundeberg et al 
(1984), Cagnie et al (2013), Salari et al (2009), Ghiasi et al (2008) (Cagnie et al., 2013; Ghiasi et al., 2008; Hanten et al., 2000; Kumar et al., 2015; Lundeberg et al., 1984; Salari, 2009). Self-myofascial tissue release as one of the corrective exercises by increasing endorphins and serotonin hormone secretion may be effective in reducing muscle pain. Another reason of effectiveness of corrective exercises can be noted the quickly selected higher than the pain sensory. Messages transmitted through corrective exercises faster transmitted to the brain and causes the release of serotonin and blocking the pain signals to the spinal cord at the beginning point of arrival. Generally, two main ways of treating trigger points, are increase local blood flow and increase the length of the sarcomere. Self-myofascial release tissue, destroys muscle tonus caused by nerve impulses coming from the spinal cord to the muscles and leads to increase blood circulation in the muscles and capillaries and ultimately reduces pain. As well as selected corrective exercises has impact on the range of motion of the neck in women lifesavers suffering from myofascial pain syndrome and forward head. As has been noted in recent researches, the effects of corrective exercises the muscles of the body can mention be more active motor units, increase blood circulation, increase the size, strength, endurance and muscle endurance in the face of external pressure and improve muscle elasticity9.results of present study are consistent with findings of Morimoto et al (2000), Ylinen et al (2007), Salari et al (2009) and Ghiasi et al (2008) (Ghiasi et al., 2008; Morimoto et al., 2000; Salari, 2009; Ylinen et al., 2007). Various studies have shown that the range of motion in people with neck trigger points are lower than healthy people and the pain leads to restrict movement. Required to achieve the desired maximum range of motion is that Antagonist muscles would be relaxed to the joint allowed to move. If there is pain, muscle tension around the joint and is needed more than the optimum stress. So the muscles become sensitive to stretch and do not allow reaching a maximum range of motion. According to the corrective exercises cause to reduce pain, this causes of decrease pain the muscles to release tension and allow more movement in the joint. On the other hand, given that the most important benefit of stretching exercises is improving joint range of motion of joint and increasing elasticity, it seems that the reason for increasing neck motion range in various direction after involving people in combined training, Considering proper stretching exercises as part of a training program in this study. In addition, selected corrective exercises can cause negative effects on the dynamics of motion neck. Because in addition to relieving pain, improve range of motion in the neck. Another reason is that the selected corrective exercises could be looking at the physiological effects of massage can offer relief from stiffness and muscle spasms in the neck muscle trigger points. As a result of these changes increases range of motion.

Results indicated that 8 weeks selected corrective training has impact on motor range of shoulder joint of women suffering from forward head posture and myofascial pain. These findings are consistent with results of Lucas (2004), Ashraf et al (2008), Kamali et al (2012), Cagnie et al (2013), Ylinen et al (2007), Salari et 
al (2012) and Ghiasi et al (2008) (Ashraf et al., 2008; Cagnie et al., 2013; Ghiasi et al., 2008; Kamali, 2010; Lucas et al., 2004; Salari, 2009; Ylinen et al., 2007).

It was seen in a research that conducted by Mohammad et al (2013) that existence an active trigger points in the muscle, causing the muscle to enter with dely. In other words, in the muscle with active trigger points, caused inhibition hence the upper trapezius muscle in healthy individuals for the movement comes into play. This action may be to increase the plate area to rotate the arm. Because according to the perspective of Moore (1992), the upward scapular rotation muscles, includes all parts of the trapezius muscle and the lower part of the anterior muscles. When the upper trapezius muscle is active trigger points, almost comes into play when the arm began to move from the side of the body but when the muscle is no active trigger point, in particular, to move the arm from the body is activated.

One of the goals of earlier activity of the upper trapezius at the initial stage of getting up arm is starting get up Akhromi joint through Acromioclavicular joint to increase space under Akhromi. The presence of trigger points in the muscle is associated with delay. That is sort of inhibition process occurs, resulting in inefficient and later the muscle activity during movement, leads to an increase in the potential for entrapping the structures under Akhromi. The results showed that self-myofascial tissue release and pull the trigger points has a positive effect such a change process and its negative effects on the movements of the shoulder joint and neck. Corrective exercises can improve muscle elasticity, which in turn can better strength and endurance due to the processes involved in. It can be stated that self-myofascial release technique is non-invasive and without side effects induced relaxation in the affected person and if it should be taught to patients and other individuals, certainly a lot of their problems and the need to reduce consumption of painkillers and sedatives.

\section{Conclusion}

Generally, it can be concluded from this study that while there are the different ways to improve the conditions of patients with trigger points but the process of corrective exercises can cause negative effects on the movements of the shoulder joint and neck because in addition to relieving pain, improve joint range of motion in the neck and shoulder patients. Therefore, it is recommended that corrective training methods be used rather than those invasive procedures that have used to treatment these people. 


\section{Author contribution}

MT performed data acquisition, data analysis; BBM performed designed the study, data analysis and manuscript preparation; AR performed data acquisition, and manuscript preparation. All authors approved the manuscript. 


\section{References}

Alter, M. (1996). Science of flexibility . Champaign, IL: Human Kinetics (google books). Amanolahi Asadollah, H.M.T., Shamsedini Ali Reza (2009). The effect of acupuncture on shoulder trigger points in patients with muscle pain syndrome. Journal of Veterinary Medicine 2, 22-34.

Ashraf, A., Mirshams, S., Salavati, A., and Yazdani, A. (2008). A comparison of the stretching with ethyl chloride spray and stretching after injection of the lidocaine in the treatment of the trigger points: single blind randomized clinical trial (RCT).

Cagnie, B., Dewitte, V., Coppieters, I., Van Oosterwijck, J., Cools, A., and Danneels, L. (2013). Effect of ischemic compression on trigger points in the neck and shoulder muscles in office workers: a cohort study. Journal of manipulative and physiological therapeutics 36, 482-489.

Ghiasi, F., Akbari, A., and Abed, M. (2008). Comparison of muscle energy techniques with ultrasound therapy in myofascial trigger point treatment in upper trapezius. Journal of Babol University of Medical Sciences 10, 7-14.

Hanten, W.P., Olson, S.L., Butts, N.L., and Nowicki, A.L. (2000). Effectiveness of a home program of ischemic pressure followed by sustained stretch for treatment of myofascial trigger points. Physical therapy 80, 997-1003.

Hodi, F.S., O'Day, S.J., McDermott, D.F., Weber, R.W., Sosman, J.A., Haanen, J.B., Gonzalez, R., Robert, C., Schadendorf, D., Hassel, J.C., et al. (2010). Improved survival with ipilimumab in patients with metastatic melanoma. The New England journal of medicine 363, 711-723.

Hou, C.-R., Tsai, L.-C., Cheng, K.-F., Chung, K.-C., and Hong, C.-Z. (2002). Immediate effects of various physical therapeutic modalities on cervical myofascial pain and trigger-point sensitivity. Archives of physical medicine and rehabilitation 83, 1406-1414.

Kamali, F.A.a.-A.S., Sara; Evangelism; Maryam; Shams Salehi, Somayeh (2010). Comparison of therapeutic effect of ischemic pressure technique on tracheal ulcer muscle in the normal position and posture muscle elasticities in computer users. Research in rehabilitation sciences 10, 56-69.

Kumar, G.Y., Sneha, P., and Sivajyothi, N. (2015). Effectiveness of Muscle energy technique, Ischaemic compression and Strain counterstrain on Upper Trapezius Trigger Points: A comparative study. International Journal of Physical Education, Sports and Health 1, 22-26.

Lucas, K.R., Polus, B.I., and Rich, P.A. (2004). Latent myofascial trigger points: their effects on muscle activation and movement efficiency. Journal of Bodywork and Movement Therapies 8, 160-166.

Lundeberg, T., Nordemar, R., and Ottoson, D. (1984). Pain alleviation by vibratory stimulation. Pain 20, 25-44.

Luo, X., Pietrobon, R., Sun, S.X., Liu, G.G., and Hey, L. (2004). Estimates and patterns of direct health care expenditures among individuals with back pain in the United States. Spine 29, 79-86.

Morimoto, M., Kawata, K., Tsuchiya, N., Murakami, H., Kura, M., and Koga, Y. (2000). [A case of acupuncture needle dermatitis]. Masui The Japanese journal of anesthesiology $49,887-889$. 
Naroii, S., Akbari, A., Asad, M., and Farahani, A. (2010). Comparing the effects of vibration and ultrasound waves accompanied with stretching exercises on myofascial trigger points of posterior neck muscles in athletes. Journal of Shahrekord University of Medical Sciences 12, 43-52.

Roach, S., Sorenson, E., Headley, B., and San Juan, J.G. (2013). Prevalence of myofascial trigger points in the hip in patellofemoral pain. Archives of physical medicine and rehabilitation 94, 522-526.

Salari, s., pilevarzadeh motahhare, M, Shafi'i, Nematollahzadeh (2009). Myofascial trigger point massage effect of short-term indices of the relaxation response. Sabzevar Faculty of Medical Sciences 11, 45-60.

Schleip, R. (2003). Fascial plasticity-a new neurobiological explanation Part 2. Journal of Bodywork and movement therapies 7, 104-116.

Shahi moridi, D., eghbali, Mansouri vaziri nejad, Reza; Najafzadeh, Neda (2009). The effect of low level laser therapy in the treatment of trapezius muscle trigger points. Mayvfasyal Journal of Medical Sciences 8, 99-108.

Timmons, B.H., and Ley, R. (1994). Behavioral and psychological approaches to breathing disorders (Springer Science \& Business Media).

Ylinen, J., Kautiainen, H., Wirén, K., and Häkkinen, A. (2007). Stretching exercises vs manual therapy in treatment of chronic neck pain: a randomized, controlled cross-over trial. Journal of rehabilitation medicine 39, 126-132. 